\section{- OPEN ACCESS}

\title{
Increased ectodomain shedding of lung epithelial cell adhesion molecule 1 as a cause of increased alveolar cell apoptosis in emphysema
}

\author{
Takahiro Mimae, ${ }_{1}^{1,2}$ Man Hagiyama, ${ }^{3}$ Takao Inoue, ${ }^{3}$ Azusa Yoneshige, ${ }^{3}$ Takashi Kato, ${ }^{3}$ \\ Morihito Okada, ${ }^{1}$ Yoshinori Murakami, ${ }^{2}$ Akihiko Ito ${ }^{3}$
}

\begin{abstract}
- Additional material is published online only. To view please visit the journal online (http://dx.doi.org/10.1136/ thoraxjnl-2013-203867)

${ }^{1}$ Department of Surgical Oncology, Research Institute for Radiation Biology and Medicine, Graduate School of Biomedical Sciences, Hiroshima University, Hiroshima, Japan ${ }^{2}$ Division of Molecular Pathology, Department of Cancer Biology, Institute of Medical Science, University of Tokyo, Tokyo, Japan ${ }^{3}$ Department of Pathology, Faculty of Medicine, Kinki University, Osaka, Japan
\end{abstract}

\section{Correspondence to} Dr A Ito, Department of Pathology, Faculty of Medicine, Kinki University, 377-2 Ohno-Higashi, Osaka-Sayama, Osaka 589-8511, Japan; aito@med.kindai.ac.jp

TM and $\mathrm{MH}$ contributed equally to this work

Received 15 May 2013 Revised 22 August 2013 Accepted 11 September 2013 Published Online First 3 October 2013

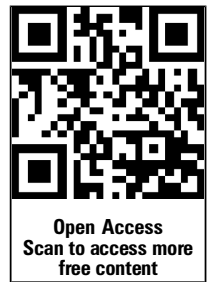

To cite: Mimae $T$, Hagiyama $M$, Inoue $T$, et al. Thorax 2014;69:223-231.

\section{ABSTRACT}

Rationale Alveolar epithelial cell apoptosis and protease/antiprotease imbalance based proteolysis play central roles in the pathogenesis of pulmonary emphysema but molecular mechanisms underlying these two events are not yet clearly understood. Cell adhesion molecule 1 (CADM1) is a lung epithelial cell adhesion molecule in the immunoglobulin superfamily. It generates two membrane associated $C$ terminal fragments (CTFs), $\alpha \mathrm{CTF}$ and $\beta C T F$, through protease mediated ectodomain shedding.

Objective To explore the hypothesis that more CADM 1-CTFs are generated in emphysematous lungs through enhanced ectodomain shedding, and cause increased apoptosis of alveolar epithelial cells.

Methods and results Western blot analyses revealed that CADM1-CTFs increased in human emphysematous lungs in association with increased ectodomain shedding. Increased apoptosis of alveolar epithelial cells in emphysematous lungs was confirmed by terminal nucleotide nick end labelling (TUNEL) assays. NCI-H441 lung epithelial cells expressing mature CADM1 but not CTFs were induced to express $\alpha$ CTF both endogenously (by shedding inducers phorbol ester and trypsin) and exogenously (by transfection). Cell fractionation, immunofluorescence, mitochondrial membrane potentiometric JC-1 dye labelling and TUNEL assays revealed that CADM1- $\alpha$ CTF was localised to mitochondria where it decreased mitochondrial membrane potential and increased cell apoptosis. A mutation in the intracytoplasmic domain abrogated all three abilities of $\alpha C T F$.

Conclusions CADM1 ectodomain shedding appeared to cause alveolar cell apoptosis in emphysematous lungs by producing $\alpha$ CTF that accumulated in mitochondria. These data link proteolysis to apoptosis, which are two landmark events in emphysema.

\section{INTRODUCTION}

Emphysema is a pulmonary disease characterised by alveolar wall destruction, resulting in enlarged airspaces and loss of surface area for gas exchange without fibrosis. ${ }^{1}$ This unique aspect of alveolar destruction has long been ascribed mainly to excessive apoptosis of alveolar structural (noninflammatory) cells (ie, epithelial and endothelial cells), and a relative excess of proteases creating a local imbalance between proteases and antiproteases. $^{2}{ }^{3}$ Apoptosis of endothelial cells in the alveolar wall is well explained by two mechanisms:

\section{Key messages}

What is the key question?

- Increased alveolar epithelial cell apoptosis and protease/antiprotease imbalance based proteolysis are two landmarks in the pathogenesis of pulmonary emphysema. What mechanisms may underlie these two events?

What is the bottom line?

- Lung epithelial cell adhesion molecule 1 (CADM1) generates two membrane associated $C$ terminal fragments (CTFs), $\alpha$ CTF and $\beta C T F$, through protease mediated ectodomain shedding. CADM1- $\alpha$ CTF were increased in human emphysematous lungs, and appeared to cause alveolar epithelial cell apoptosis by localising to mitochondria and decreasing mitochondrial membrane potential.

\section{Why read on?}

- This study identifies CADM1- $\alpha$ CTF as a key molecule responsible for linking between proteolysis and apoptosis in emphysematous lungs, and will aid the development of a target based therapeutic strategy for emphysema.

decreased maintenance signals for endothelial cells mediated through vascular endothelial growth factor and its cognate receptor, and increased proteolysis of extracellular matrices in the alveolar wall resulting from a protease/antiprotease imbalance. ${ }^{3} 4$ However, the molecular basis for alveolar epithelial cell apoptosis specific to emphysema is not yet fully understood. Involvement of a protease/antiprotease imbalance has generally been speculated because degradation of the extracellular matrix caused by excessive proteases forces alveolar cells to fall into anoikis, a type of programmed cell death, secondary to cell detachment from the matrix. ${ }^{4}$ However, studies over the past decade have suggested that alveolar epithelial destruction in emphysematous lungs might occur due to apoptosis, possibly unrelated to matrix degradation induced by proteases. An in vitro experiment showed that leucocyte elastase induces apoptosis in lung epithelial cells by changing mitochondrial permeability, mediated by a protease activated receptor 1 triggered pathway 
involving activation of nuclear factor $\mathrm{\kappa B}$ and $\mathrm{p} 53 .^{5}$ Cathepsin $\mathrm{S}$, a cysteine proteinase secreted from pulmonary macrophages, mediates alveolar epithelial cell apoptosis in interferon $\gamma$ induced emphysema of mice by activating both mitochondrial and death receptor pathways. ${ }^{6}$ Matrix metalloproteinases trigger activation of the death receptor apoptotic pathway by processing the tumour necrosis factor $\alpha$ precursor and Fas ligand to yield their bioactive forms. ${ }^{78}$ These results suggest that excessive proteases can directly act on alveolar epithelial cells and cause apoptosis, but this possibility has not been intensively examined.

Cell adhesion molecule 1 (CADM1), also widely known as tumour suppressor in lung cancer 1 (TSLC1), is an intercellular adhesion molecule in the immunoglobulin superfamily. It is a membrane spanning glycoprotein composed of three extracellular immunoglobulin-like domains, a single transmembrane region and a short carboxy terminal intracytoplasmic tail with a protein 4.1 interaction sequence and a PDZ type II domain binding motif. ${ }^{9}$ CADM1 localises to the lateral plasma membrane in pulmonary and biliary epithelia and binds transhomophilically between adjacent cells. ${ }^{10}{ }^{11}$ Consequently, it is assumed to contribute to the integrity of epithelial cell structure and polarity. ${ }^{12}$ Recent studies by our own and other laboratories have revealed that CADM1 expression is regulated by posttranscriptional mechanisms, including glycosylation and proteolytic cleavage, called shedding. ${ }^{13}{ }^{14}$ CADM1 is cleaved at two sites in its ectodomain, yielding two membrane associated $\mathrm{C}$ terminal fragments (CTF), termed $\alpha \mathrm{CTF}$ and $\beta \mathrm{CTF}$. This ectodomain shedding appears to occur on the plasma membrane because this event proceeds in isolated plasma membranes and is directly mediated by a membrane bound metalloprotease called a disintegrin and metalloproteinase 10 (ADAM10). ${ }^{14}$ These CTFs are subsequently cleaved within the plasma membrane by $\gamma$ secretase, yielding an intracellular domain (ICD). ${ }^{14}$ Although we previously proposed CADM1 shedding as a candidate mechanism for downregulating full length CADM1, ${ }^{14}$ it remains unknown whether the products generated by shedding (ie, CTFs and ICD) have any biological function.

In the present study, we compared CADM1 expression between emphysematous and normal lungs and found that $\alpha \mathrm{CTF}$ and $\beta \mathrm{CTF}$ increased in emphysematous lungs in association with increased ectodomain shedding of CADM1. Because alveolar cell apoptosis also increased in emphysematous lungs, we then examined the possible association between CADM1 ectodomain shedding and alveolar cell apoptosis. Human lung epithelial cells were induced to express endogenous $\alpha \mathrm{CTF}$ by shedding inducers and were transfected to express exogenous $\alpha \mathrm{CTF}$. Cell fractionation and immunofluorescence experiments revealed that $\alpha \mathrm{CTF}$ localised to mitochondria. This localisation appeared to result in mitochondrial depolarisation and induction of cell apoptosis. These data identify CADM1- $\alpha$ CTF as a key molecule that links two landmark events in emphysema, proteolysis and apoptosis.

\section{MATERIALS AND METHODS}

All materials and methods used in this study are described in detail in the online supplementary methods.

\section{RESULTS}

\section{Increased shedding of CADM1 in emphysematous lungs}

Surgically resected lungs were examined histologically by pathologists (see online supplementary figure S1), and 10 normal subjects and 11 patients with emphysematous lungs were enrolled. Patient characteristics are summarised in table 1. The histological diagnosis was consistent with the results of preoperative respiratory function tests, except that three subjects with normal lungs (case Nos 5, 8 and 9) had low carbon monoxide transfer factor (Tlco) and one patient with emphysematous lungs (case No 13) had fairly good forced expiratory volume at $1 \mathrm{~s}\left(\mathrm{FEV}_{1}\right) /$ forced vital capacity (FVC) and Tlco. All patients with emphysematous lungs were cigarette smokers, as revealed by their Brinkman Indices (table 1). Based on this observation and our previous finding that smoking may alter CADM1 expression in the lung, ${ }^{15}$ we subgrouped subjects with normal lungs into smokers $(n=5)$ and non-smokers $(n=5)$. The lung tissue lysates were analysed by western blotting with a polyclonal antibody raised against the CADM1 C terminal 15 amino acid peptide. CADM1 $\alpha C T F, \beta C T F$ and ICD were recognisable by this antibody. The full length form of CADM1 and its two shed forms, $\alpha \mathrm{CTF}$ and $\beta \mathrm{CTF}$, were detected at about 100,20 and $35 \mathrm{kDa}$, respectively (figure 1A). Bands detected at 50 and $25 \mathrm{kDa}$ corresponded to the non-glycosylated full length form and $\beta C T F$, respectively, as we reported previously. ${ }^{11}$ The expression level of the full length form normalised to $\beta$-actin decreased significantly in emphysematous lungs compared with normal lungs (figure 1B). This might be attributable to a low content of epithelial cells in the tissue lysates because emphysematous lungs have a lower number of alveolar epithelial cells, the major source of CADM1 in the peripheral lung. ${ }^{10}$ This speculation was supported by a western blot reprobed with an antibody against E-cadherin, an epithelial cell marker, ${ }^{10}$ which revealed that the full length form of CADM1 and E-cadherin levels were well correlated in normal $\left(R^{2}=0.734 ; \mathrm{p}<0.001\right)$ and emphysematous $\left(R^{2}=0.586 ; \mathrm{p}<0.001\right)$ lungs (see online supplementary figure S2).

Table 1 Clinical characteristics of patients with normal and emphysematous lungs

\begin{tabular}{|c|c|c|c|c|c|c|c|c|c|c|c|c|c|c|c|c|c|c|c|c|c|}
\hline \multirow[b]{2}{*}{ Case } & \multicolumn{5}{|c|}{ Normal (non-smoker) } & \multicolumn{5}{|c|}{ Normal (smoker) } & \multicolumn{11}{|c|}{ Emphysema (smoker) } \\
\hline & 1 & 2 & 3 & 4 & 5 & 6 & 7 & 8 & 9 & 10 & 11 & 12 & 13 & 14 & 15 & 16 & 17 & 18 & 19 & 20 & 21 \\
\hline Age (years) & 64 & 50 & 48 & 63 & 76 & 60 & 56 & 80 & 71 & 84 & 79 & 62 & 62 & 68 & 76 & 58 & 80 & 70 & 70 & 84 & 79 \\
\hline Sex & $\mathrm{F}$ & $M$ & $M$ & $\mathrm{~F}$ & $\mathrm{~F}$ & $M$ & $\mathrm{~F}$ & $M$ & $M$ & $M$ & $M$ & $M$ & $M$ & $M$ & $M$ & $\mathrm{~F}$ & $\mathrm{~F}$ & $M$ & M & $M$ & $M$ \\
\hline Brinkman Index & 0 & 0 & 0 & 0 & 0 & 900 & 500 & 2400 & 1000 & 2220 & 980 & 800 & 1600 & 1600 & 1000 & 600 & 200 & 1000 & 1000 & 1280 & 1200 \\
\hline Cause of surgery & $A D$ & $A D$ & $A D$ & $A D$ & $A D$ & $A D$ & $A D$ & SCLC & SQ & $A D$ & SQ & $A D$ & $A D$ & PC & $A D$ & $A D$ & $A D$ & SCLC & $\mathrm{CH}$ & SQ & $A D$ \\
\hline Excised lung lobe* & $\mathrm{RL}$ & RU & $\mathrm{RU}$ & RU & $\mathrm{LL}$ & $\mathrm{RU}$ & $\mathrm{RL}$ & $\mathrm{RU}$ & $\mathrm{RL}$ & LU & $\mathrm{RU}$ & $\mathrm{RU}$ & RU & LU & LU & $\mathrm{RU}$ & $\mathrm{RL}$ & $\mathrm{RU}$ & RM & $\mathrm{RU}$ & $\mathrm{RL}$ \\
\hline $\mathrm{FEV}_{1} / \mathrm{FVC}$ & 82.3 & 80.5 & 86.6 & 86.8 & 79.9 & 73.0 & 76.7 & 76.6 & 78.1 & 84.1 & 61.7 & 73.2 & 75.1 & 55.0 & 88.8 & 58.1 & 74.6 & 76.7 & 73.0 & 83.5 & 61.5 \\
\hline Tlco (\%) & 101.8 & NE & 92.8 & 76.5 & 49.3 & 102.0 & 81.2 & 57.0 & 41.2 & 89.3 & 37.8 & $\mathrm{NE}$ & 71.2 & NE & 44.4 & 36.8 & 55.3 & 54.8 & 53.0 & 36.3 & 68.2 \\
\hline
\end{tabular}

$A D$, adenocarcinoma; $C H$, chondroid hamartoma; $F_{E V}$, forced expiratory volume in $1 \mathrm{~s}$; FVC, forced vital capacity; NE, not examined; PC, plemorphic carcinoma; SCLC, small cell lung 
A

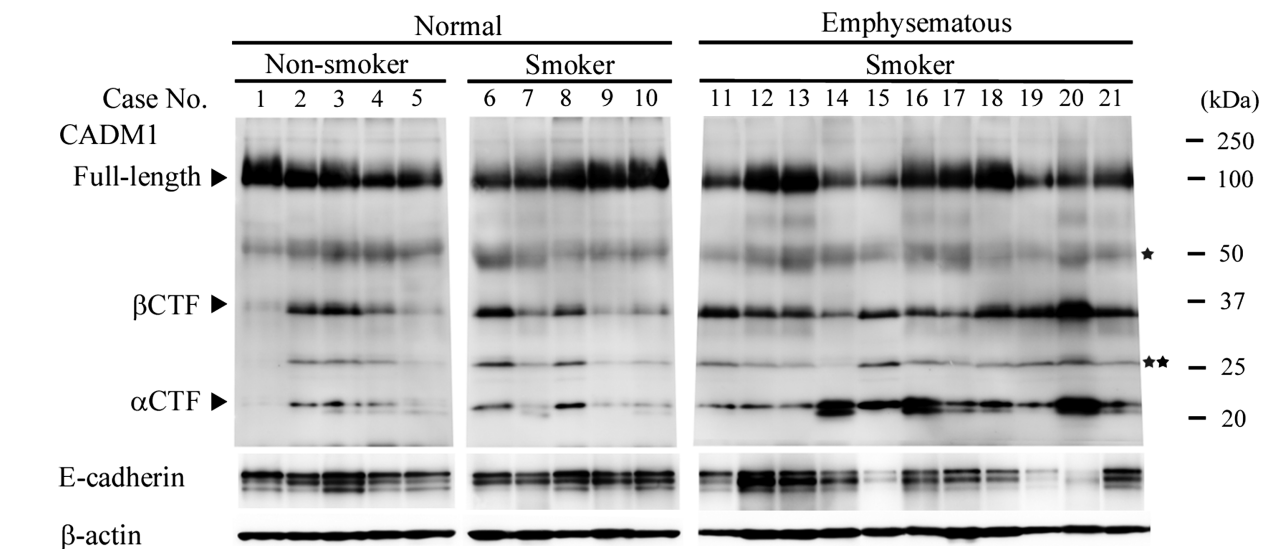

B

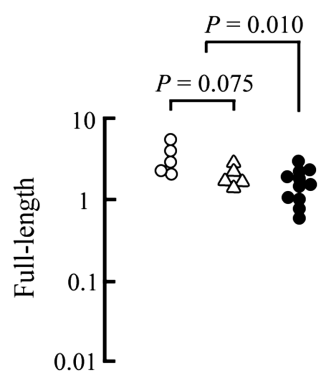

$\mathrm{C}$
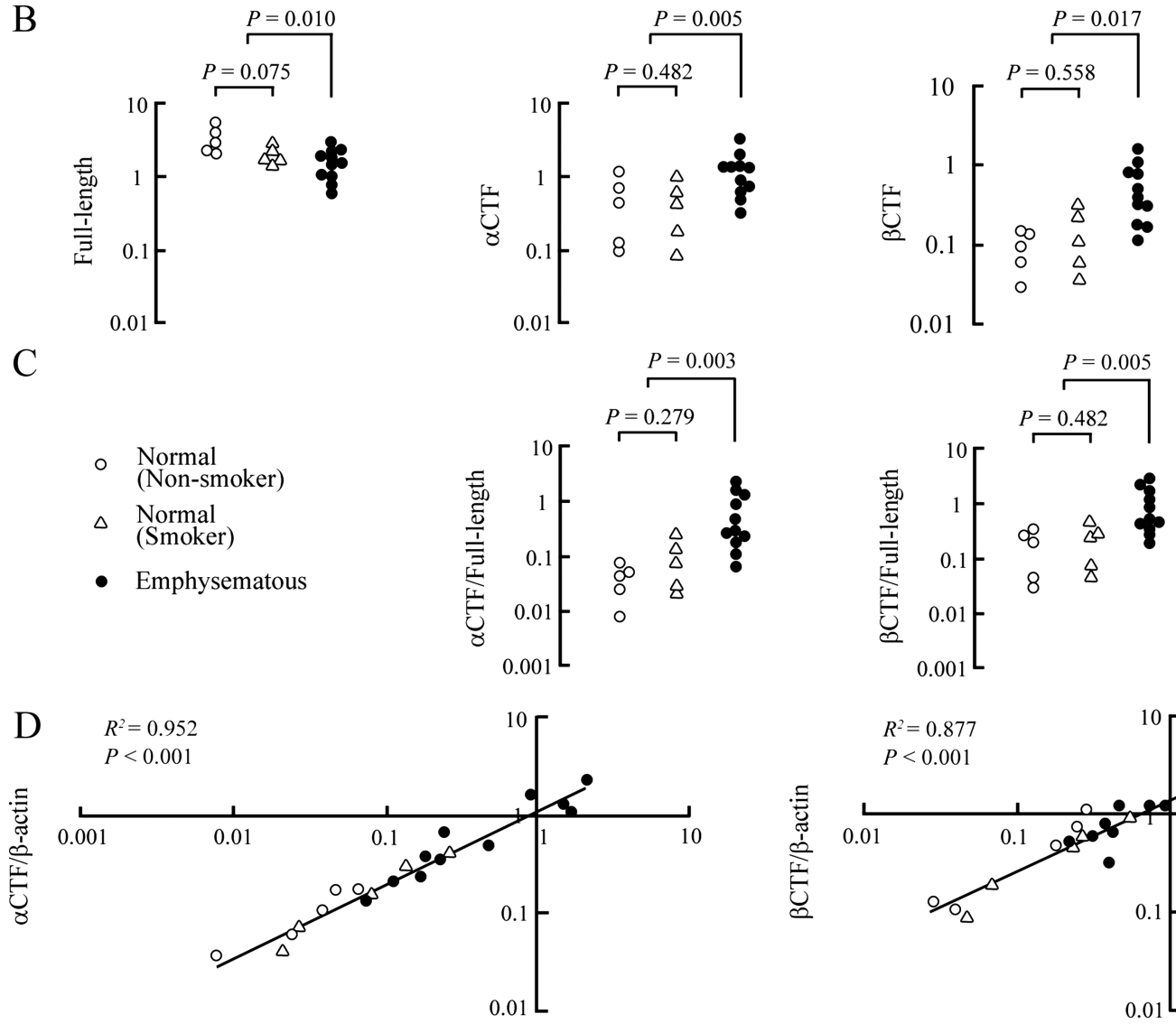

$\alpha \mathrm{CTF} /$ Full-length

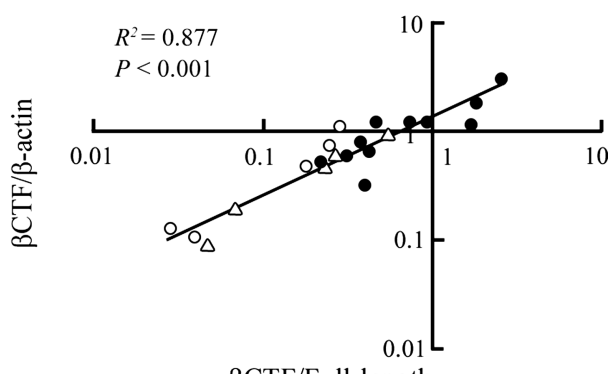

$\beta C T F / F u l l-l e n g t h$

Figure 1 Increased ectodomain shedding of cell adhesion molecule 1 (CADM1) in emphysematous lungs. (A) Western blot analyses of CADM1 and E-cadherin in normal and emphysematous lungs. Cases are numbered as in table 1. Bands corresponding to the non-glycosylated full length form and $\beta C$ terminal fragment (CTF) are depicted by one and two asterisks, respectively. The blots were reprobed with an anti- $\beta$-actin antibody to indicate protein loading per lane. (B) Graphs plotted with dots indicating relative expression levels of CADM1 molecules. In each lane of (A), intensities of bands specific to CADM1 full length form, $\alpha C T F$ and $\beta C T F$, and $\beta$-actin were quantified using NIH ImageJ software, and the intensities of CADM1 molecules were normalised to $\beta$-actin. Statistical significance was analysed by the Mann-Whitney $U$ test, and $p$ values are shown. (C) Graphs plotted with dots indicating expression levels of $\alpha C T F$ and $\beta C T F$ relative to the full length form of CADM1. Statistical significance was analysed by the Mann-Whitney $U$ test, and $p$ values are shown. (D) Graphs with $X$ and $Y$ axes in band intensity ratios. On the left, $\alpha C T F / f u l l$ length and $\alpha C T F / \beta$-actin were plotted on the $X$ and $Y$ axes, respectively. On the right, $\beta C T F / f u l l$ length and $\beta C T F / \beta$-actin were plotted on the $X$ and $Y$ axes, respectively. In each graph, the two ratios were well approximated as linear. Correlations and statistical significance were analysed by Spearman's rank test, and $R^{2}$ and $\mathrm{p}$ values are shown.

In contrast, expression levels of $\alpha \mathrm{CTF}$ and $\beta \mathrm{CTF}$ increased significantly in emphysematous lungs, while they were comparable between smokers and non-smokers with normal lungs (figure 1B). We also calculated the signal intensity ratios of $\alpha \mathrm{CTF}$ and $\beta \mathrm{CTF}$ to the full length form for each case, and found that these ratios were significantly higher in emphysematous lungs than those in normal lungs (figure 1C), and were strongly positively correlated with $\alpha \mathrm{CTF}$ and $\beta \mathrm{CTF}$ levels $\left(R^{2}=0.952\right.$ and $0.877 ; \mathrm{p}<0.001$ ), respectively (figure $1 D)$. The ratios of the two shed forms were comparable between smokers and non-smokers with normal lungs. These results indicate that CADM1 ectodomain shedding was 
accelerated to generate more $\alpha \mathrm{CTF}$ and $\beta \mathrm{CTF}$ in emphysematous lungs.

\section{Increased apoptosis of alveolar cells in emphysematous lungs}

Lung sections were double stained by the terminal deoxynucleotidyl transferase mediated dUTP nick end labelling (TUNEL; green) and E-cadherin immunofluorescence (red) (figure 2A, B). Alveolar epithelial cells were identified by membranous staining for E-cadherin. Practically all alveolar cells were TUNEL negative in normal lungs, irrespective of smoking habit, whereas $>10 \%$ of alveolar cells on average were TUNEL positive in emphysematous lungs $(\mathrm{p}<0.002)$ (figure $2 \mathrm{C}$ ).

\section{CADM1- $\alpha$ CTF localises to mitochondria}

To probe for a possible link between increased CADM1 ectodomain shedding and increased alveolar cell apoptosis, we used NCI-H441 cells, a human lung epithelial cell line with characteristics of Clara cells. Western blot analyses detected abundant expression of the full length form of CADM1 in NCI-H441 cells grown under standard culture conditions, but the two shed forms $\alpha \mathrm{CTF}$ and $\beta C T F$ were undetectable, indicating that CADM1 was rarely shed in steady state NCI-H441 cells. We used phorbol 12-myristate 13-acetate (PMA) and trypsin to induce CADM1 shedding, which both induce ectodomain shedding of transmembrane proteins. ${ }^{14}{ }^{16}$ When the cells were treated with a mixture of PMA $(200 \mathrm{nM})$ and trypsin $(0.0125 \%$ $\mathrm{w} / \mathrm{v}$, a concentration low enough to prevent cell detachment) for $20 \mathrm{~min}$, but not with either alone, a considerable amount of $\alpha \mathrm{CTF}$ appeared with a slight decrease in the amount of full length CADM1, indicating that CADM1 ectodomain shedding was induced by the treatment (figure 3). The reason why $\beta C T F$ was not produced by the treatment is unknown. Considering that $\beta$ shedding of $\beta$-amyloid precursor protein, a key event in Alzheimer's disease, occurs within cells, ${ }^{17}$ trypsin may have no potential to activate $\beta$ shedding due to its inaccessibility to $\beta$-sheddase(s).

PMA and trypsin treated or untreated cells were then double stained by CADM1 immunofluorescence and Mitotracker dye, a mitochondrial marker. CADM1 immunostaining was exclusively
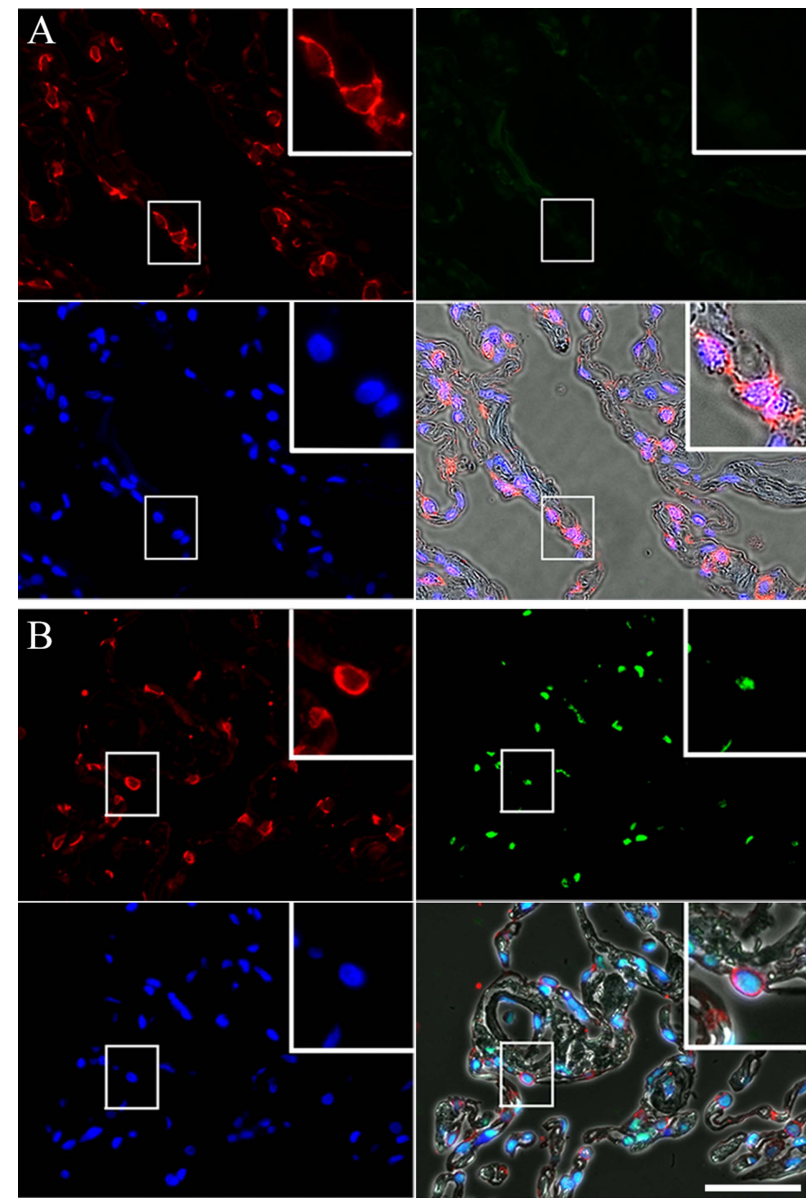

$\mathrm{C}$
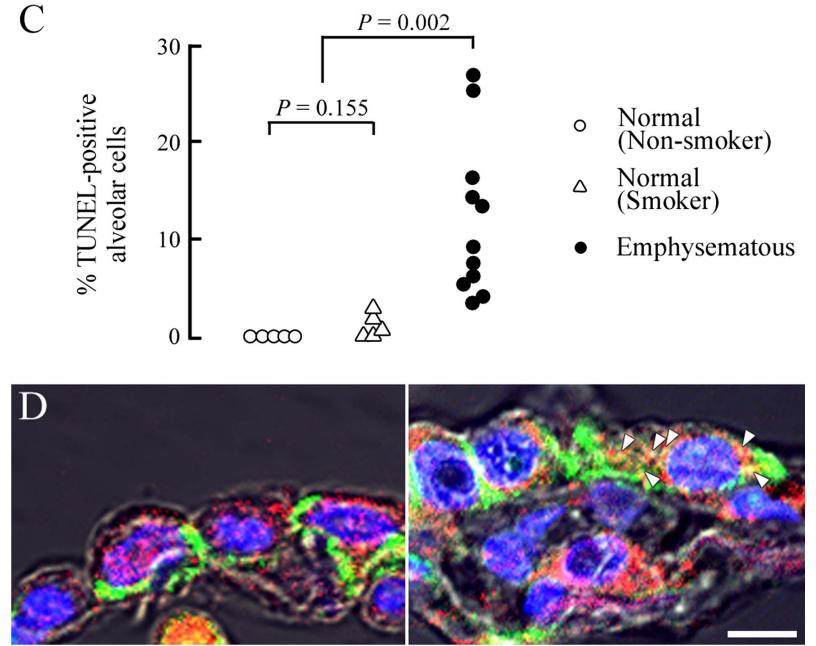

Figure 2 Increased apoptosis of alveolar epithelial cells in emphysematous lungs. (A, B) Representative results of terminal deoxynucleotidyl transferase dUTP nick end labelling (TUNEL) assays for normal (A) and emphysematous (B) lungs. Formalin fixed, paraffin embedded lung sections were triple stained by E-cadherin immunofluorescence (red; left upper), the TUNEL method (green; right upper) and 4',6-diamino-2-phenylindole (DAPI, for nuclear counterstain; blue; left lower). These three images were merged on the differential interference contrast image (right lower). TUNEL negative and positive alveolar epithelial cells are enlarged in insets of (A) and (B), respectively. Bar=50 $\mu \mathrm{m}$. (C) Graph plotted with dots indicating TUNEL positive alveolar epithelial cell proportions in individual patients who were divided into two groups, normal and emphysematous lungs. The normal lung patients were further divided into non-smokers and smokers. Statistical significance was analysed between groups by the Student's t test, and $p$ values are shown. (D) Double immunofluorescence of normal (left) and emphysematous (right) lung sections for cell adhesion molecule 1 (CADM1) (green) and mitochondria (clone 113-1; red). Sections were counterstained by DAPI (blue). Three fluorescence images are merged on the differential interference contrast image. Arrowheads point to examples of colocalisation of CADM1 and mitochondrial immunostaining. Bar=10 $\mu \mathrm{m}$. 


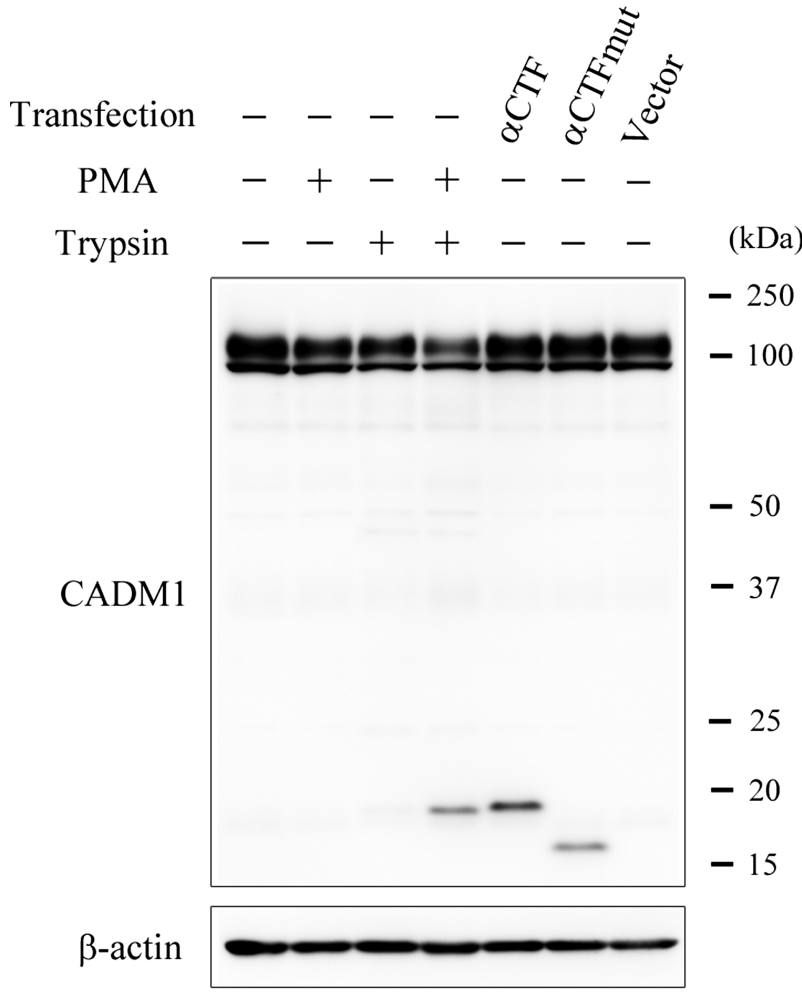

Figure 3 Induction of cell adhesion molecule 1 (CADM1) ectodomain shedding and exogenous expression of $\alpha \mathrm{C}$ terminal fragment (CTF) and $\alpha \mathrm{CTFmut}$ in $\mathrm{NCl}-\mathrm{H} 441$ cells. $\mathrm{NCl}-\mathrm{H} 441$ cells were treated with either phorbol myristic acid (PMA $200 \mathrm{nM})$ or trypsin $(0.0125 \% \mathrm{w} / \mathrm{v})$, or a mixture of both, or were transfected with pCX4bsr-SP- $\alpha C T F$, pCX4bsr-SP- $\alpha C T F m u t$ or an empty vector. After 20 min of treatment and 2 days of transfection, the cells were subjected to western blot analyses with the CADM1 antibody.

detected on the cell membrane of untreated cells (figure 4Aa), whereas PMA and trypsin treatment resulted in a marked appearance of cytoplasmic stains for CADM1, which were significantly colocalised with Mitotracker stain (figure 4Ab, B), suggesting subcellular localisation of CADM1- $\alpha \mathrm{CTF}$ to mitochondria.

We examined this possibility by expressing $\alpha \mathrm{CTF}$ exogenously. Because CADM1 ectodomain shedding likely occurs on the cell surface, ${ }^{14} \alpha \mathrm{CTF}$ should be primarily a transmembrane protein. Thus according to our previous mass spectrometric data that determined the $\mathrm{N}$ terminal amino acid residue of $\alpha \mathrm{CTF},{ }^{14}$ we constructed a plasmid vector expressing a large deletion form of CADM1, in which the signal peptide was ligated upstream of $\alpha \mathrm{CTF}$ (pCX4bsr-SP- $\alpha \mathrm{CTF}$ ). We mutated the intracytoplasmic domain of $\alpha \mathrm{CTF}$ as a control so that the resulting domain would not target to mitochondria but to the cytosol (pCX4bsr-SP- $\alpha$ CTFmut; online supplementary figure S3), according to an intracellular localisation prediction algorithm (WoLF PSORT; refer to online supplementary methods). We transfected NCI-H441 cells with either plasmid construct, and 2 days later confirmed that the transfectants expressed a considerable amount of exogenous $\alpha \mathrm{CTF}$ or $\alpha \mathrm{CTFmut}$ by western blot analyses (figure 3). The ratios of $\alpha \mathrm{CTF}$ to full length CADM1 in transfectants were equivalent to those in emphysematous lungs (see online supplementary figure S4). Taken together with the western blot results indicating that endogenous full length CADM1 in NCI-H441 cells was certainly lower than that in normal lung epithelial cells in vivo (see online supplementary figure S4), transfectants with pCX4bsr-SP- $\alpha$ CTF were considered to resemble epithelial cells from emphysematous lungs rather than normal lungs, in terms of CADM1 protein levels.

NCI-H441 transfectants were double stained with CADM1 immunofluorescence and Mitotracker dye. Transfectants with pCX4bsr-SP- $\alpha$ CTF exhibited a staining pattern quite similar to that of PMA and trypsin treated cells; the cytoplasmic CADM1 signals were well colocalised with Mitotracker stain (figure 4Ac, B). In contrast, pCX4bsr-SP- $\alpha$ CTFmut transfectants showed a strong CADM1 membranous staining with weak but significant cytoplasmic signals that were rarely colocalised with Mitotracker stain (figure 4Ad, B).

Mitochondrial localisation of $\alpha \mathrm{CTF}$ was examined by cell fractionation of NCI-H441 cells expressing endogenous and exogenous $\alpha \mathrm{CTF}$. Exogenous $\alpha \mathrm{CTF}$ and $\alpha \mathrm{CTFmut}$ were expressed as $\mathrm{N}$ terminally FLAG tagged forms to clearly distinguish them from endogenous $\alpha \mathrm{CTF}$ using the p3xFLAG-CMV-9 vector, which is designed to deliver the protein encoded by the cDNA insert efficiently to the cell surface. Expression of FLAG tagged proteins was confirmed by western blotting with an anti-FLAG antibody (see online supplementary figure S5). Transfected or untransfected NCI-H441 cells were treated with a mixture of PMA $(200 \mathrm{nM})$ and trypsin $(0.25 \% \mathrm{w} / \mathrm{v})$ for 20 min to induce endogenous $\alpha \mathrm{CTF}$ and detach cells without mitochondrial damage and then were fractionated into cytosolic and mitochondrial fractions. Whole cytoplasmic lysates without nuclei were also prepared from aliquots of the treated cells. Successful fractionation was verified by western blotting analyses, showing enrichment of glyceraldehyde 3-phosphate dehydrogenase (G3PDH), a cytosolic marker, and cytochrome c oxidase subunit IV (CoxIV), a mitochondrial marker, in the corresponding fractions (figure 5). Reprobing with the CADM1 antibody revealed that both endogenous and exogenous $\alpha \mathrm{CTF}$ were detected exclusively in the mitochondrial fraction as CoxIV, whereas $\alpha C T F m u t$ was enriched in the cytosolic fraction as greatly as G3PDH (figure 5).

Lung sections were double stained with antibodies against CADM1 and mitochondria. CADM1 immunostaining in alveolar epithelial cells was primarily membranous in normal lungs whereas it was occasionally both membranous and cytoplasmic in emphysematous lungs and cytoplasmic staining was appreciably colocalised with mitochondrial staining (figure 2D). The proportion of epithelial cells with this colocalisation signal was significantly larger in emphysematous lungs $(n=4)$ than in normal lungs $(n=4)(11.3 \pm 8.3$ vs $1.8 \pm 1.5 \% ; p=0.038)$.

\section{CADM1- $\alpha$ CTF decreases mitochondrial membrane potential and induces apoptosis}

We examined whether CADM1- $\alpha \mathrm{CTF}$ might alter mitochondrial membrane potential $(\Delta \Psi \mathrm{m})$ that normally exists across the inner mitochondrial membrane using the JC-1 probe, a lipophilic cationic dye that exhibits $\Delta \Psi \mathrm{m}$ dependent accumulation in mitochondria, as indicated by a fluorescence shift from green $(\sim 525 \mathrm{~nm})$ to red $(\sim 590 \mathrm{~nm})$. NCI-H441 transfectants expressing exogenous $\alpha \mathrm{CTF}$ or $\alpha \mathrm{CTFmut}$ were stained with JC- 1 dye at 24, 48 and $72 \mathrm{~h}$ post-transfection, and mitochondrial depolarisation was assessed by measuring the red/green fluorescence intensity ratio of the dye (figure $6 \mathrm{~A}$ and see online supplementary figure S6). $\alpha$ CTFmut did not change the JC-1 ratio at any time point, whereas $\alpha \mathrm{CTF}$ significantly decreased the ratio at 48 and $72 \mathrm{~h}$ (figure $6 \mathrm{~B}$ ). Transfected and untransfected NCI-H441 cells were stained by the TUNEL technique $48 \mathrm{~h}$ after transfection. $\alpha \mathrm{CTFmut}$ did not change the proportion 
A $\quad$ CADM1 1
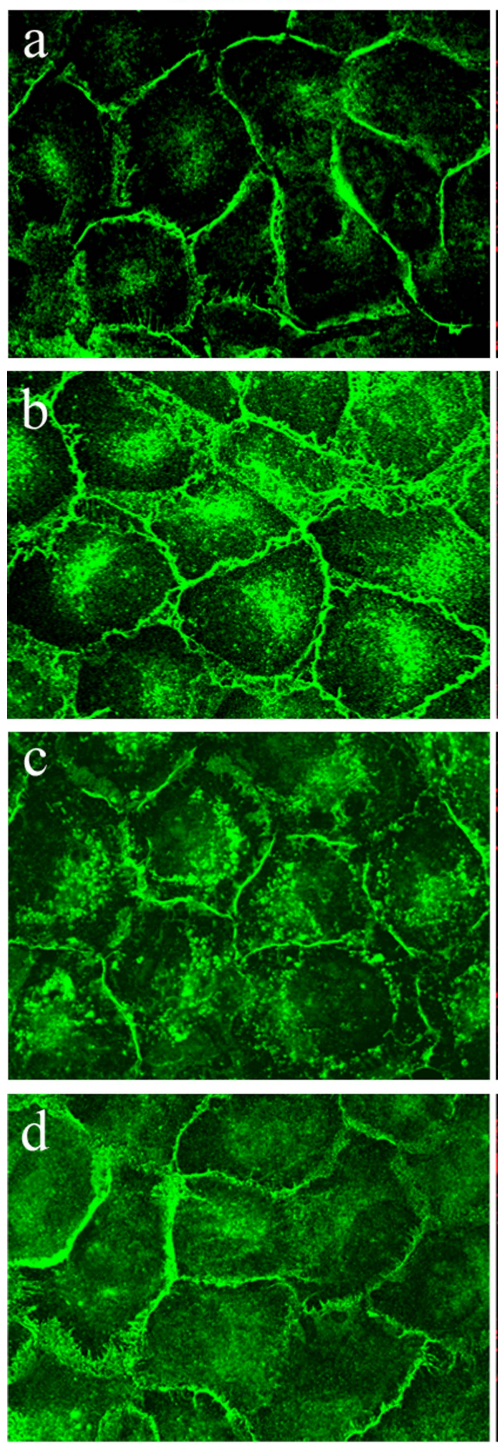

Mitotracker
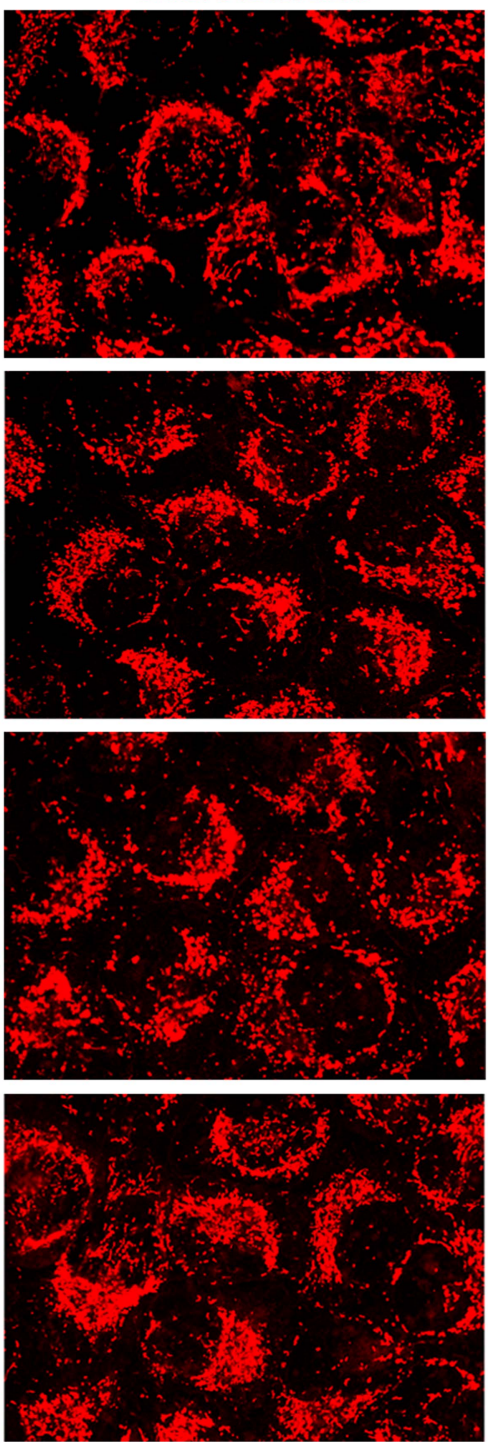

Merge
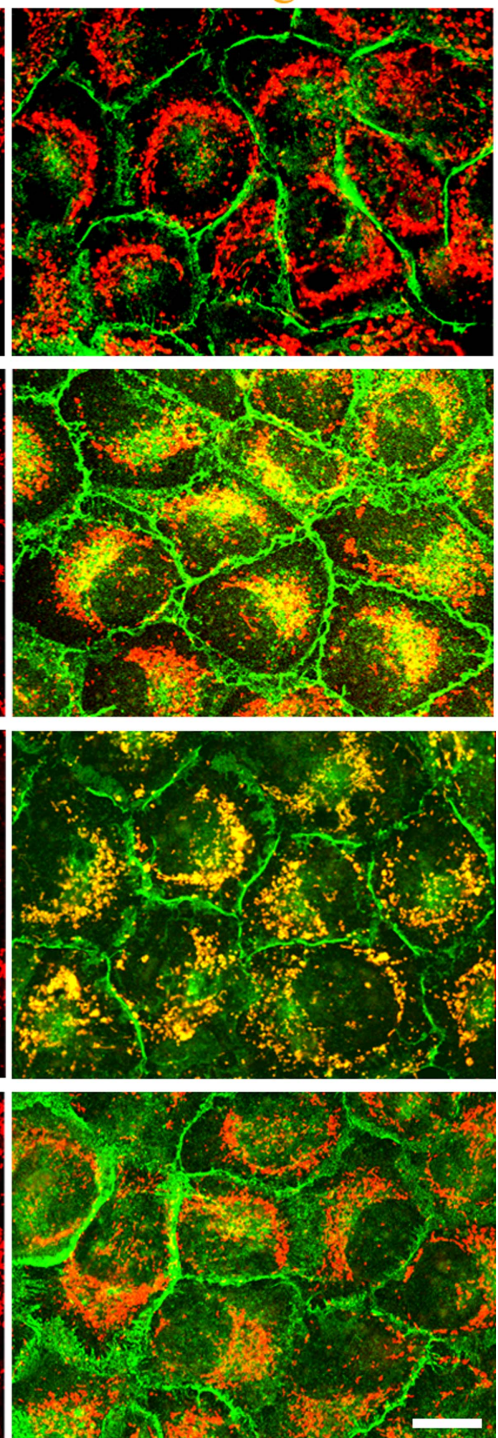

B

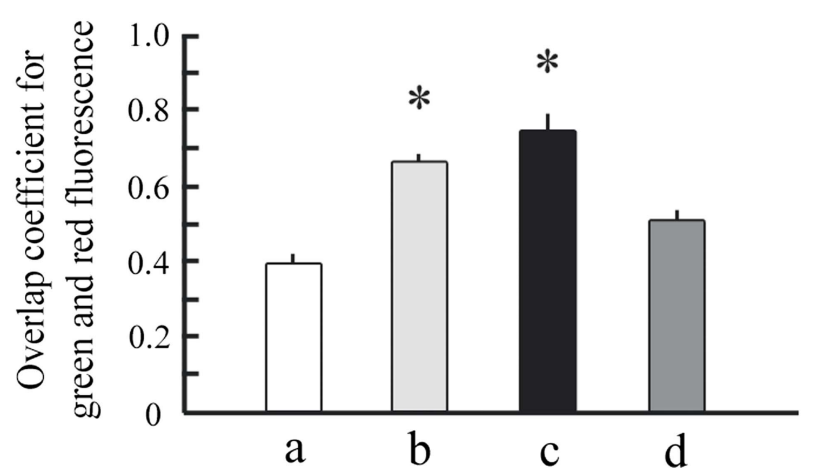

Figure 4 Immunofluorescence of cell adhesion molecule 1 (CADM1) with Mitotracker staining in $\mathrm{NCl}$-H441 cells expressing $\alpha \mathrm{C}$ terminal fragment (CTF) and $\alpha$ CTFmut. (A) NCI-H441 cells were untreated (a) or treated with a mixture of phorbol myristic acid and trypsin (b), or were transfected with pCX4bsr-SP- $\alpha C T F$ (c) or pCX4bsr-SP- $\alpha C T F m u t$ (d). Then, cells were double stained with CADM1 immunofluorescence (green; left) and Mitotracker fluorescence (red; middle). In the merged images (right), yellow areas mean colocalisation of both fluorescent signals - that is, mitochondrial localisation of CADM1. Bar=10 $\mu \mathrm{m}$. (B) Graph showing overlap coefficients in NCl-H441 cells of the four types (a-d shown in (A)). Intensity correlation between green and red fluorescence was quantified using ImageJ Colocalisation Analysis, and overlap coefficients were calculated. Data are expressed as mean $\pm S D$, and statistical significance was analysed by the Student's $t$ test. ${ }^{*} p<0.01$ compared with the value of untreated cells $(a$ in $(A))$. 


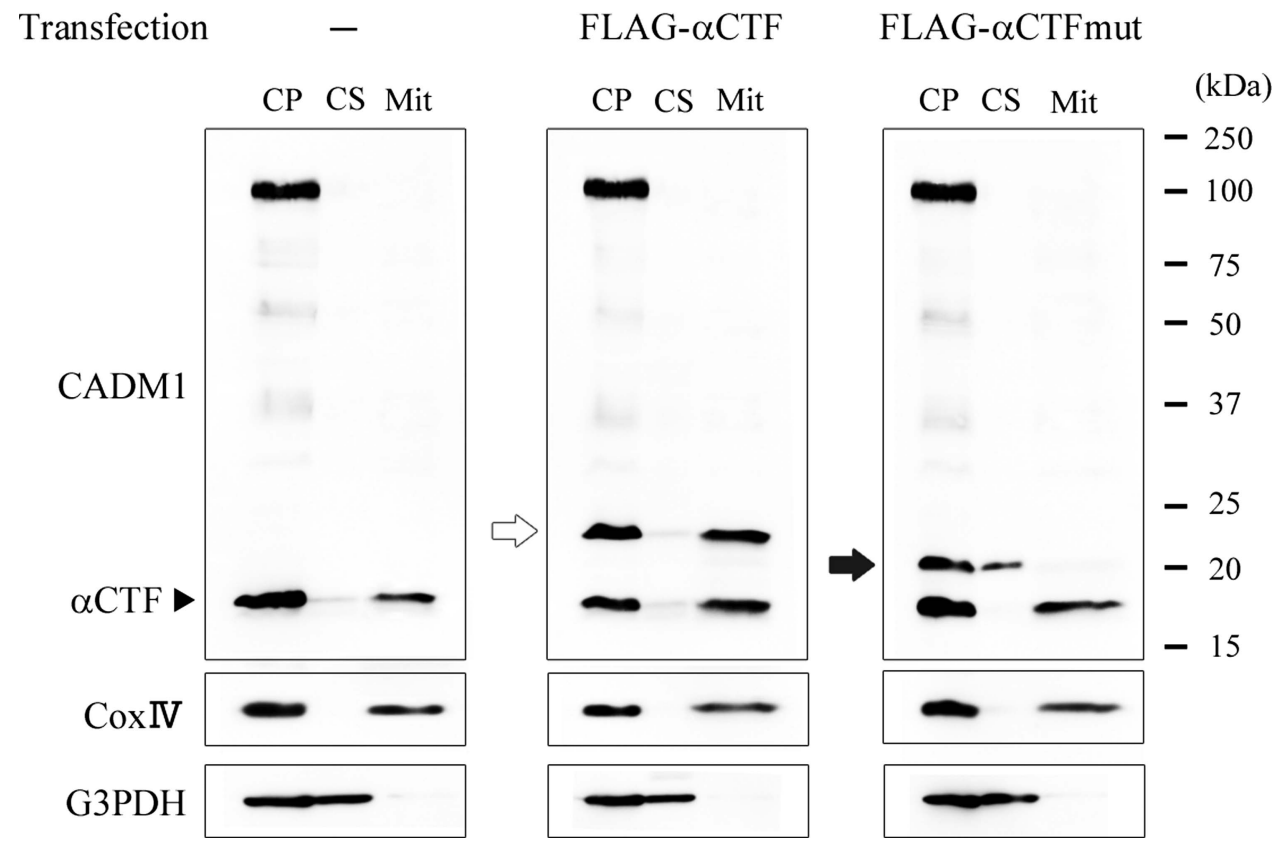

Figure 5 Cell fractionation experiments of $\mathrm{NCl}-\mathrm{H} 441$ cells expressing $\alpha \mathrm{C}$ terminal fragment (CTF) and $\alpha \mathrm{CTFmut}$. NCI-H441 cells were untransfected (left) or transfected with p3xFLAG- $\alpha$ CTF (middle) or p3xFLAG- $\alpha$ CTFmut (right), and were fractionated into cytosolic (CS) and mitochondrial (Mit) fractions. Whole cytoplasmic lysates (CP) were extracted from aliquots of the cells. These lysates and fractions were analysed with western blotting using antibodies against cell adhesion molecule 1 (CADM1), cytochrome c oxidase subunit IV (CoxIV) and glyceraldehyde 3-phosphate dehydrogenase (G3PDH). Open and closed arrows indicate FLAG tagged $\alpha$ CTF and $\alpha C T F m u t$, respectively.

of TUNEL positive cells whereas $\alpha \mathrm{CTF}$ increased the proportion fivefold $(\mathrm{p}<0.01)$ (figure $6 \mathrm{~B})$.

\section{DISCUSSION}

We found that CADM1 ectodomain shedding increased in emphysematous lungs from smoking patients, but not in normal lungs from smoking patients, suggesting that oxidants in cigarette smoke may act as a critical inducer of CADM1 ectodomain shedding only in subjects who have particular genetic backgrounds. Of interest, changes in emphysema susceptible genes, such as $\alpha-1$ antitrypsin, ${ }^{18}$ macrophage elastase, ${ }^{19}$ klotho $^{20}$ and surfactant $\mathrm{D},{ }^{21}$ lead to a relative excess of proteases, creating a local protease/antiprotease imbalance. ${ }^{19}{ }^{21-23}$ In the present study, all patients with smoking habits were obliged to quit smoking more than 1 month before the date of surgery. Therefore, oxidants seem not to promote CADM1 shedding through its direct ongoing action but rather seem to help establish long lasting protease/antiprotease imbalances in alveoli.

All but one patient (case No 19) analysed in this study had lung cancer. Because CADM1 is known to be downregulated in lung cancer due to promoter methylation, ${ }^{24}$ these patients had potentially impaired CADM1 expression even in non-cancerous lungs. We performed western blot analyses of a small number of emphysematous lungs that did not develop lung cancer, and detected a relative increase in $\alpha \mathrm{CTF}$ and $\beta \mathrm{CTF}$ to full length CADM1 (see online supplementary figure S7). Increased amounts of $\alpha \mathrm{CTF}$ and $\beta C T F$ appeared to be present in emphysematous lungs as a result of increased ectodomain shedding of CADM1 in emphysematous lungs, both with and without lung cancer.

Cell fractionation and immunofluorescence experiments consistently showed that $\alpha \mathrm{CTF}$ localised to mitochondria. This finding appeared to be relevant in vivo, as we detected intracytoplasmic CADM1 that was associated with mitochondria in alveolar epithelial cells from emphysematous lungs (figure 2D).
Mutagenesis experiments revealed a decisive role for the intracytoplasmic domain in this subcellular localisation. How the intracytoplasmic domain leads $\alpha \mathrm{CTF}$ to mitochondria remains to be addressed. A growing body of evidence is accumulating to show that cell membrane spanning proteins, such as epidermal growth factor receptor and mucin 1, can translocate to mitochondria. ${ }^{25} 26$ Although mechanisms underlying these events remain largely unknown, clathrin mediated endocytosis is shown to be involved. ${ }^{26}$ After internalisation, mucin 1 is assumed to utilise heat shock proteins as molecular chaperons for mitochondrial translocation. ${ }^{25}$ Higashiyama et al demonstrated that the remnant peptides generated by ectodomain shedding of type I integral membrane proteins, such as pro-heparin binding epidermal growth factor-like growth factor and pro-amphiregulin, are internalised into endocytotic vesicles. ${ }^{27} 28$ The $\mathrm{N}$ and $\mathrm{C}$ termini of the peptides are positioned inside and outside of the vesicles, respectively, and the $\mathrm{C}$ terminal tail, free in the cytosol, plays a decisive role in the intracellular destinations of the remnant peptide. ${ }^{27} 28 \alpha \mathrm{CTF}$ may be present as a vesicle associated transmembrane molecule in the cytoplasm, with its $\mathrm{C}$ terminal tail being free outside the vesicle, and this $\mathrm{C}$ terminal tail may carry a conformational signal that serves as a binding site for molecular chaperons, such as heat shock protein family members.

Exogenous $\alpha \mathrm{CTF}$ decreased mitochondrial membrane potential in NCI-H441 cells and increased apoptosis, suggesting that mitochondrial localisation of $\alpha \mathrm{CTF}$ might result in activation of the mitochondrial apoptosis pathway. Mao et al reported that exogenous CADM1 induces caspase 3 activation and apoptosis in A549 lung adenocarcinoma cells lacking endogenous CADM1, and that protein 4.1 binding motif and PDZ domain binding motif in the intracytoplasmic domain are indispensable for this induction. ${ }^{29}$ Members of the membrane associated guanylate kinase (MAGuK) family are known as binding partners to the latter motif. ${ }^{30}$ Interestingly, this family contains a subgroup that carries the caspase recruitment domain in its $\mathrm{N}$ terminal 


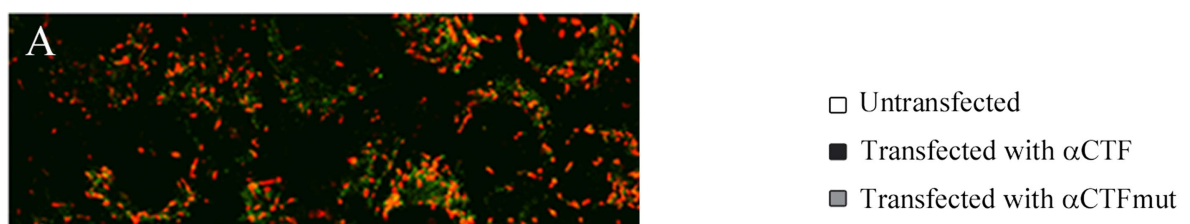

$\mathrm{B}$

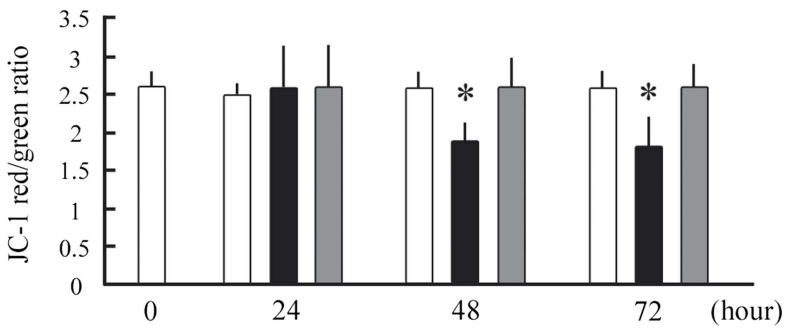

$\mathrm{C}$

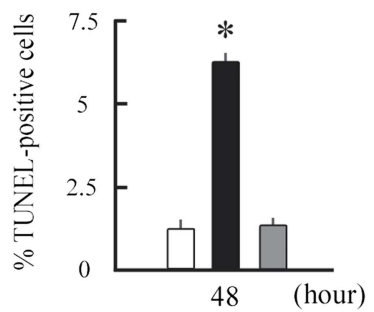

Figure $6 \alpha \mathrm{C}$ terminal fragment (CTF) decreases mitochondrial membrane potential in NCI-H441 cells and increases apoptosis. (A) Representative results of JC-1 staining in NCl-H441 cells. NCl-H441 cells were untransfected (upper) or transfected with pCX4bsr-SP- $\alpha$ CTF (middle) or pCX4bsr-SP- $\alpha$ CTFmut (lower), and were stained $48 \mathrm{~h}$ later with JC-1 dye. Images were captured by a confocal laser microscope, and green and red fluorescence signals were merged. Differential interference contrast images are shown in online supplementary figure $\mathrm{S} 6$. Bar=20 $\mu \mathrm{m}$. (B) $\mathrm{Graph}$ showing changes in JC-1 red/green ratios in $\mathrm{NCl}-\mathrm{H} 441$ cells after transfection. $\mathrm{NCl}-\mathrm{H} 441$ cells were untransfected or transfected with

pCX4bsr-SP- $\alpha C T F$ or pCX4bsr-SP- $\alpha$ CTFmut, and were stained with JC-1 dye at the indicated time points. Cells were observed through a confocal laser microscope and were morphometrically analysed to calculate JC-1 red/green ratios. Data are expressed as mean \pm SD, and statistical significance was analysed by the Student's test. ${ }^{*} p<0.01$ compared with the value of untransfected cells. (C) Graph showing the proportion of terminal deoxynucleotidyl transferase dUTP nick end labelling (TUNEL) positive $\mathrm{NCl}-\mathrm{H} 441$ cells at 2 days after transfection. Cells were transfected as in (B). After 2 days, cells were stained with the TUNEL method, and the proportions of TUNEL positive cells were calculated. Data are expressed as mean $\pm S D$, and statistical significance was analysed by the Mann-Whitney $U$ test. ${ }^{*} p<0.01$ compared with the value in untransfected cells.

region and participates in apoptosis signalling. ${ }^{31} \alpha \mathrm{CTF}$ and $\beta C T F$, which both share the intracytoplasmic domain, once produced, may activate the mitochondrial apoptosis pathway by transporting particular MAGuK family members to mitochondria in alveolar epithelial cells.

There are several splice variants of human CADM1, named isoforms SP1 to SP4. ${ }^{32}$ Reverse transcription-PCR revealed that nine lungs examined and NCI-H441 cells all expressed SP4 exclusively (see online supplementary figure S8). Tanabe et al showed that SP1 and SP2 are shed constitutively, while SP3 is non-cleavable. ${ }^{33}$ Our data proved SP4 cleavable. SP4 ectodomain shedding appeared to be not constitutive but induced by particular pathological stimuli. Moiseeva et al reported that SP4 overexpressing HMC-1 mast cells show better survival and lower caspase $3 / 7$ activity than SP1 overexpressing cells. ${ }^{34}$ This difference between two isoforms may be explained by their distinct susceptibility to ectodomain shedding. In HMC-1 cells, SP1 may produce more $\alpha \mathrm{CTF}$ and/or $\beta C T F$ than SP4, resulting in activation of the mitochondrial apoptosis pathway.
In conclusion, we propose increased ectodomain shedding of CADM1 as a novel molecular mechanism for increased alveolar cell apoptosis in emphysematous lungs. This mechanism is an extension of the conventional understanding that proteolytic activity is locally excessive in emphysematous lung alveoli because CADM1 ectodomain shedding per se is a proteolytic process, and also suggests that selective inhibitors to block CADM1 sheddase activity and/or mitochondrial localisation of CADM1 shedding products can slow or halt the progression of emphysema. In fact, ADAM10 is released by human alveolar macrophages, and intratracheal administration of an adenoviral vector expressing ADAM10 in mice results in the development of emphysema. ${ }^{35}$ Further characterisation of CADM1 ectodomain shedding and its associated molecular events will open a new avenue for target based therapeutic approaches to emphysema.

Contributors Al and YM designed the study, and Al wrote the manuscript. Al, TM and $\mathrm{MO}$ provided the clinical samples. TM, MH, TI, AY and TK performed the experiments, and $\mathrm{MH}$ analysed the data. 
Funding This study was supported by the Japan Society for the Promotion of Science Kakenhi (24890274 and 25860302 to MH, 24659184 to TI, 24890137 to TM, and 24590492 to Al), and grants from the Yasuda Medical Foundation and the Osaka Medical Research Foundation for Intractable Diseases.

\section{Competing interests None.}

Patient consent Obtained.

Ethics approval Ethics approval was provided by the ethics committee of Hiroshima University and Kinki University, Japan (approval Nos Eki-350 and 25-088).

Provenance and peer review Not commissioned; externally peer reviewed.

Open Access This is an Open Access article distributed in accordance with the Creative Commons Attribution Non Commercial (CC BY-NC 3.0) license, which permits others to distribute, remix, adapt, build upon this work non-commercially, and license their derivative works on different terms, provided the original work is properly cited and the use is non-commercial. See: http://creativecommons.org/ licenses/by-nc/3.0/

\section{REFERENCES}

1 Snider GL, Kleinerman J, Thurlbeck WM, et al. The definition of emphysema. Report of a National Heart, Lung, and Blood Institute, Division of Lung Diseases workshop. Am Rev Respir Dis 1985;132:182-85.

2 Tuder RM, Petrache I, Elias JA, et al. Apoptosis and emphysema: the missing link. Am J Respir Cell Mol Biol 2003;28:551-4.

3 Taraseviciene-Stewart L, Voelkel NF. Molecular pathogenesis of emphysema. J Clin Invest 2008;118:394-402.

4 Demedts IK, Demoor T, Bracke KR, et al. Role of apoptosis in the pathogenesis of COPD and pulmonary emphysema. Respir Res 2006;7:53.

5 Suzuki T, Yamashita C, Zemans RL, et al. Leukocyte elastase induces lung epithelial apoptosis via a PAR-1-, NF-kappaB-, and p53-dependent pathway. Am J Respir Cell Mol Biol 2009;41:742-55

6 Zheng T, Kang MJ, Crothers K, et al. Role of cathepsin S-dependent epithelial cell apoptosis in IFN-gamma-induced alveolar remodeling and pulmonary emphysema. J Immunol 2005:174:8106-15.

7 Powell WC, Fingleton $\mathrm{B}$, Wilson $\mathrm{CL}$, et al. The metalloproteinase matrilysin proteolytically generates active soluble Fas ligand and potentiates epithelial cell apoptosis. Curr Biol 1999;9:1441-7.

8 Mohan MJ, Seaton T, Mitchell J, et al. The tumor necrosis factor-alpha converting enzyme (TACE): a unique metalloproteinase with highly defined substrate selectivity. Biochemistry 2002;41:9462-9.

9 Kuramochi M, Fukuhara $\mathrm{H}$, Nobukuni T, et al. TSLC1 is a tumor-suppressor gene in human non-small-cell lung cancer. Nat Genet 2001;27:427-30.

10 Ito A, Okada M, Uchino K, et al. Expression of the TSLC1 adhesion molecule in pulmonary epithelium and its down-regulation in pulmonary adenocarcinoma other than bronchioloalveolar carcinoma. Lab Invest 2003:83:1175-83.

11 Ito A, Nishikawa Y, Ohnuma K, et al. SgIGSF is a novel biliary-epithelial cell adhesion molecule mediating duct/ductule development. Hepatology 2007:45:684-94

12 Sakurai-Yageta M, Masuda M, Tsuboi Y, et al. Tumor suppressor CADM1 is involved in epithelial cell structure. Biochem Biophys Res Commun 2009;390:977-82.

13 Fogel Al, Li Y, Giza J, et al. N-glycosylation at the SynCAM (synaptic cell adhesion molecule) immunoglobulin interface modulates synaptic adhesion. J Biol Chem 2010;285:34864-74.

14 Nagara $Y$, Hagiyama $M$, Hatano N, et al. Tumor suppressor cell adhesion molecule 1 (CADM1) is cleaved by a disintegrin and metalloprotease 10 (ADAM10) and subsequently cleaved by gamma-secretase complex. Biochem Biophys Res Commun 2012:417:462-7.
15 Kikuchi S, Yamada D, Fukami T, et al. Hypermethylation of the TSLC1/IGSF4 promoter is associated with tobacco smoking and a poor prognosis in primary nonsmall cell lung carcinoma. Cancer 2006;106:1751-8.

16 Ding K, Lopez-Burks M, Sanchez-Duran JA, et al. Growth factor-induced shedding of syndecan-1 confers glypican-1 dependence on mitogenic responses of cancer cells. J Cell Biol 2005;171:729-38.

17 Chyung AS, Greenberg BD, Cook DG, et al. Novel beta-secretase cleavage of beta-amyloid precursor protein in the endoplasmic reticulum/intermediate compartment of NT2N cells. J Cell Biol 1997;138:671-80.

18 Martorana PA, Brand T, Gardi C, et al. The pallid mouse. A model of genetic alpha 1-antitrypsin deficiency. Lab Invest 1993;68:233-41.

19 Hautamaki RD, Kobayashi DK, Senior RM, et al. Requirement for macrophage elastase for cigarette smoke-induced emphysema in mice. Science 1997:277:2002-4.

20 Kuro-o M, Matsumura Y, Aizawa $\mathrm{H}$, et al. Mutation of the mouse klotho gene leads to a syndrome resembling ageing. Nature 1997;390:45-51.

21 Wert SE, Yoshida M, LeVine AM, et al. Increased metalloproteinase activity, oxidant production, and emphysema in surfactant protein $D$ gene-inactivated mice. Proc Natl Acad Sci U S A 2000:97:5972-7.

22 Kidokoro Y, Kravis TC, Moser KM, et al. Relationship of leukocyte elastase concentration to severity of emphysema in homozygous alpha1-antitrypsin-deficient persons. Am Rev Respir Dis 1977;115:793-803.

23 Funada Y, Nishimura Y, Yokoyama M. Imbalance of matrix metalloproteinase-9 and tissue inhibitor of matrix metalloproteinase- 1 is associated with pulmonary emphysema in Klotho mice. Kobe J Med Sci 2004:50:59-67.

24 Fukami T, Fukuhara $\mathrm{H}$, Kuramochi $\mathrm{M}$, et al. Promoter methylation of the TSLC1 gene in advanced lung tumors and various cancer cell lines. Int J Cancer 2003;107:53-9.

25 Ren J, Bharti A, Raina D, et al. MUC1 oncoprotein is targeted to mitochondria by heregulin-induced activation of c-Src and the molecular chaperone HSP9O. Oncogene 2006;25:20-31.

26 Demory ML, Boerner JL, Davidson $\mathrm{R}$, et al. Epidermal growth factor receptor translocation to the mitochondria: regulation and effect. J Biol Chem 2009:284:36592-604.

27 Hieda M, Isokane M, Koizumi M, et al. Membrane-anchored growth factor, HB-EGF on the cell surface targeted to the inner nuclear membrane. J Cell Biol 2008;180:763-9.

28 Isokane M, Hieda M, Hirakawa S, et al. Plasma-membrane-anchored growth factor pro-amphiregulin binds A-type lamin and regulates global transcription. J Cell Sci 2008;121:3608-18.

29 Mao X, Seidlitz E, Truant R, et al. Re-expression of TSLC1 in a non-small-cell lung cancer cell line induces apoptosis and inhibits tumor growth. Oncogene 2004;23:5632-42.

30 Murakami Y. Involvement of a cell adhesion molecule, TSLC1/IGSF4, in human oncogenesis. Cancer Sci 2005:96:543-52.

31 Bertin J, Wang L, Guo Y, et al. CARD11 and CARD14 are novel caspase recruitment domain (CARD)/membrane-associated guanylate kinase (MAGUK) family members that interact with BCL10 and activate NF-kappa B. J Biol Chem 2001;276:11877-82.

32 Biederer T. Bioinformatic characterization of the SynCAM family of immunoglobulin-like domain-containing adhesion molecules. Genomics 2006;87:139-50.

33 Tanabe Y, Kasahara T, Momoi T, et al. Neuronal RA175/SynCAM1 isoforms are processed by tumor necrosis factor-alpha-converting enzyme (TACE)/ADAM17-like proteases. Neurosci Lett 2008;444:16-21.

34 Moiseeva EP, Leyland ML, Bradding P. CADM1 is expressed as multiple alternatively spliced functional and dysfunctional isoforms in human mast cells. Cell Mol Life Sci 2012;69:2751-64.

35 Saitoh H, Leopold PL, Harvey BG, et al. Emphysema mediated by lung overexpression of ADAM10. Clin Trans/ Sci 2009:2:50-6. 